\title{
Improving the electrical properties of carbon nanotubes with interhalogen \\ compounds
}

\author{
Dawid Janas $^{\text {a,b,* }}$, Karolina Z. Milowska ${ }^{\mathrm{a}}$, Paul D. Bristowe ${ }^{\mathrm{a}}$, Krzysztof K.K. Koziol ${ }^{\mathrm{a}}$ \\ ${ }^{a}$ Department of Materials Science and Metallurgy, University of Cambridge, 27 Charles \\ Babbage Rd, CB3 OFS Cambridge, United Kingdom \\ ${ }^{b}$ Department of Chemistry, Silesian University of Technology, B. Krzywoustego 4, 44-100 \\ Gliwice, Poland
}

\begin{abstract}
The electronic properties of carbon nanostructures such as carbon nanotubes (CNTs) or graphene can easily be tuned by the action of various doping agents. We present an experimental study and numerical analysis of how and why metallic and semiconductive CNTs can be p-doped by exposing them to two interhalogens: iodine monochloride and iodine monobromide. Simple application of these compounds was found to reduce the electrical resistance by as much as $2 / 3$ without causing any unfavorable chemical modification, which could disrupt the highly conductive network of $\mathrm{sp}^{2}$ carbon atoms. To gain better insight into the underlying mechanism of the observed experimental results, we provide a first principles indication of how interhalogens interact with model metallic $(5,5)$ and semiconductive $(10,0)$ CNTs.
\end{abstract}

Key words: carbon nanotubes; thin films; doping; electrical properties; halogens.

\section{Introduction}

Ever since their discovery, individual carbon nanotubes (CNT) have shown many remarkable properties. They are among the strongest [1, 2], thermally [3-5] and electrically [6, 7] conductive materials on Earth. However, one of the often underappreciated features is their

\footnotetext{
*Corresponding author. Tel/Fax: +44 1223334567. E-mail address: dj295@cam.ac.uk (D. Janas).
} 
tunability. CNTs can be synthesized or functionalized to have a desired set of properties. Depending on their architecture on the nanoscale, CNTs can be metallic with very high electrical conductivity [8] useful for. data/power transmission or semiconducting with an appropriate band gap for application in devices. In the latter area, CNTs have recently reached another milestone and finally outperformed silicon transistors due to quasi ballistic transport [9].

The electrical properties of CNTs are quite sensitive to the presence of doping agents. Dopants can either have a physical interaction with the CNT electronic structure or be chemically embedded into the CNT framework. In the former scenario, electron rich compounds such as alkali metals $[10,11]$ or nitrogen-compounds $[12,13]$ are used for $n$ doping, whereas electron-poor halogens [14] or mineral acid [15, 16] are employed for pdoping. In the latter scenario, the most common approach involves incorporation of boron atoms (for p-doping) or nitrogen atoms (for n-doping) during synthesis, which in addition to their influence on electrical properties, also has a significant effect on the material's nanostructure [17].

Here we present an experimental study and numerical analysis on the influence of the addition of iodine and two interhalogen compounds (iodine monochloride and iodine monobromide) on the structural and electrical properties of CNTs. Empirical data shows powerful doping of the CNT films with as much as $2 / 3$ reduction of electrical resistance, which, most importantly, is permanent. First principles calculations reveal for the first time why these compounds tune the electrical performance of CNTs to this extent.

\section{Experimental}

CNT films were produced by the direct-spinning method reported elsewhere $[18,19]$. In brief, methane was decomposed at $1200^{\circ} \mathrm{C}$ in hydrogen atmosphere. Due to the catalytic 
action of iron from ferrocene, CNT aerogel was formed from carbon feed. Thiophene was used as promoter to facilitate the synthesis and speed up the process. As-made material was spun out onto a rotating spindle where it deposited in the form of fibers, which, as the reaction time progressed, formed a continuous sheet of CNT film. Samples $10 \mathrm{~mm}$ x $40 \mathrm{~mm}$ were cut and peeled off from the support for use in the experiments.

The samples were then subjected to treatment with interhalogen compounds. They were placed in a bath filled with $1 \mathrm{M}$ solutions of iodine monochloride or iodine monobromide in dichloromethane (DCM) for 15 minutes. A sample treated with neat dichloromethane was also used as a reference. Once the samples were removed from the solutions, they were left to dry overnight in ambient conditions. Untreated samples were also used as a reference.

A number of microscopy/spectroscopy techniques were employed to characterise the samples. Scanning Electron Microscopy (SEM, FEI Nova NanoSEM) probed the microstructure and quality of the CNTs films. Raman spectroscopy (Renishaw inVia, $\lambda=633$ $\mathrm{nm})$ was used to analyse the surface chemistry of the samples. The intensity ratio $\left(\mathrm{I}_{\mathrm{D}} / \mathrm{I}_{\mathrm{G}}\right)$ of the defect-induced band (D) to the graphitic vibration band (G) indicated the degree of structural perfection of the material. For each sample, ten spectra were accumulated between 1150 and $1800 \mathrm{~cm}^{-1}$ to eliminate the influence of background. Energy-Dispersive X-ray spectroscopy (EDX, Bruker Quantax coupled with Nova NanoSEM) was used to record the elemental composition at $10 \mathrm{keV}$ emission voltage.

Thermogravimetric analysis (TGA, Mettler Toledo TGA/DSC system) was used to measure changes in chemical and physical properties with temperature. The samples were heated to $1000^{\circ} \mathrm{C}$ at a rate of $10^{\circ} \mathrm{C} \mathrm{min}^{-1}$ in a $20 \mathrm{ml} \mathrm{min}-1$ flow of air.

Resistance measurements were performed with a Keithley 2000 multimeter using a fourprobe method [20]. The $10 \mathrm{~mm}$ x $40 \mathrm{~mm}$ samples were placed onto custom-designed sample holders with electrical terminals made of copper. An average and standard deviation among 
ten samples for each treatment was determined.. Resistance was normalized to the measured initial resistance recorded at room temperature to account for variation in thickness among the samples.

\section{Computational}

The structural and electronic properties of the halogen doped CNTs were studied in the framework of the spin polarized density functional theory (DFT) [21, 22] as implemented in the SIESTA numerical package $[23,24]$. The computations which involved full geometry optimization were performed using the generalized gradient approximation (GGA) of the exchange-correlation functional with the Perdew-Burke-Ernzerhof (PBE) parametrization. The influence of the core electrons was accounted for using normconserving Troullier-Martins [26] nonlocal pseudopotentials cast in Kleinman-Bylander [27] separable form. The valence electrons were represented with double- $\zeta$ numerical orbital basis sets localized on atoms, including polarization functions. The van der Waals interaction between the halogens and the CNT were described using the long-range dispersion correction to the energy as proposed by Grimme [28]. The kinetic cut-off for real-space integrals was set to $350 \mathrm{Ry}$, whereas the Brillouin zone was sampled in the 2x2x20 Monkhorst and Pack scheme [29] For the band structure and density of states calculations, the kinetic cut-off for real-space integrals was increased to $600 \mathrm{Ry}$. The self-consistent field (SCF) cycle was iterated until the total energy changed by less than $10^{-4} \mathrm{eV} /$ atom and the density matrix by less than $10^{-4}$. The maximum force tolerance acting on the atoms equalled $0.01 \mathrm{eV} / \AA$.

To explore the influence of halogen doping on different types of CNT, we consider two model single-walled CNTs: metallic $(5,5)$ and semiconducting $(10,0)$ together with three separate halogen doping agents: $\mathrm{I}_{2}, \mathrm{ICl}$ and $\mathrm{IBr}$ (Fig. 1). Armchair and zigzag CNTs have differently oriented $\mathrm{sp}^{2}$ bonds along nanotube's symmetry axis. Therefore, in order to obtain the most energetically favourable structures, the initial orientation of the halogen molecules 
above the lateral surface of both types of CNT were chosen differently for the $(5,5)$ and $(10,0)$ types. These orientations were maintained after full geometry optimization of all the systems considered and are shown in Fig. 1.
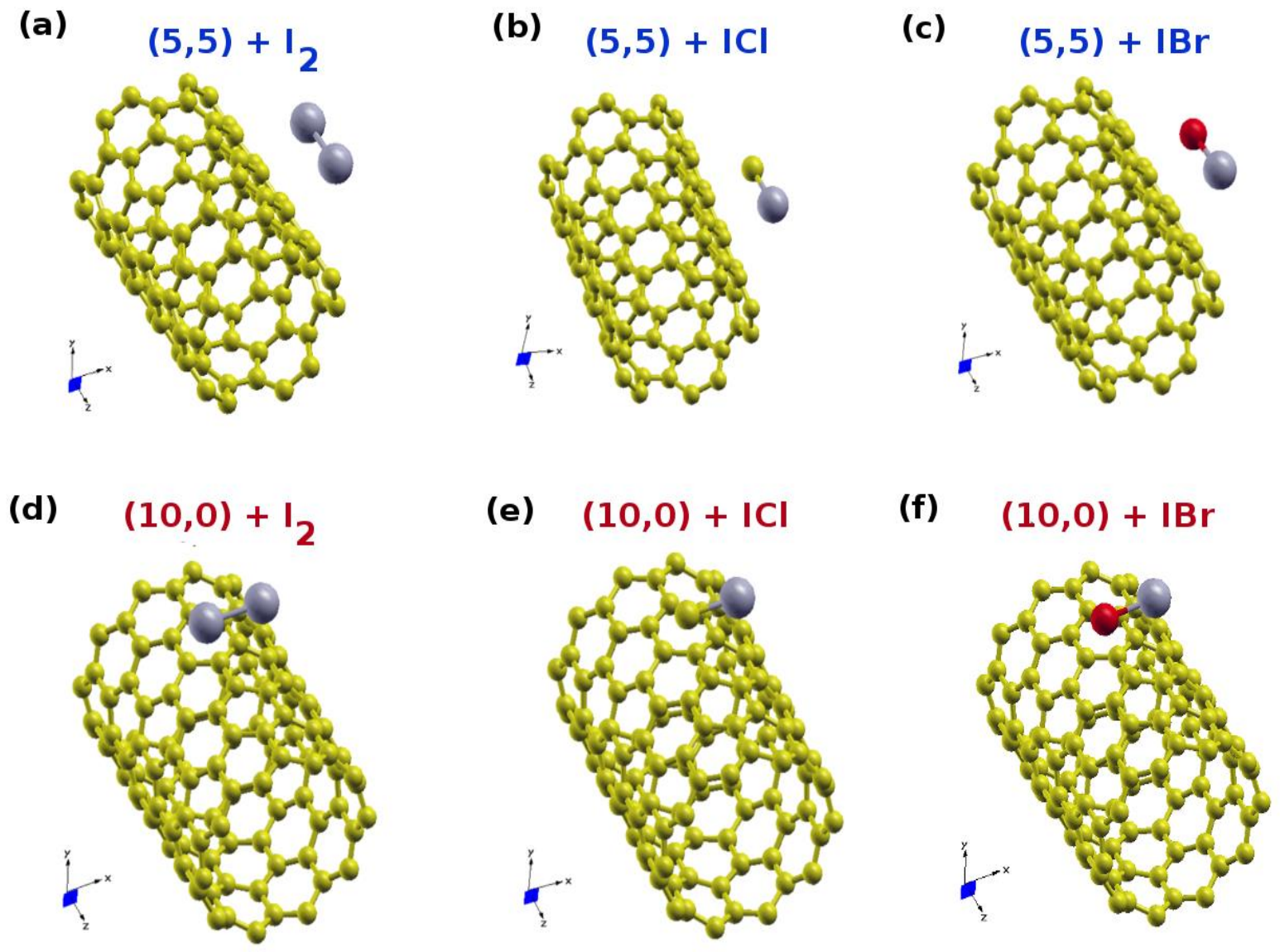

Fig. 1 The optimized structures of the $(5,5)$ and $(10,0)$ CNTs interacting with $\mathrm{I}_{2}, \mathrm{IBr}$ and ICl molecules. The $\mathrm{C}$ and $\mathrm{Cl}$ atoms are depicted in yellow using different sized spheres while I and $\mathrm{Br}$ are shown in blue and in red respectively.

All the calculations were performed using supercells with the lateral separation of CNTs equal to $20 \AA$, which is large enough to eliminate completely the spurious interaction between neighbouring cells. Also the halogen molecules were separated from each other by a distance large enough to assume they were isolated.

In order to quantify morphological changes in the CNT structure induced by functionalization, we calculated the coefficient of variation of the CNT radius (CV). This is 
defined as the ratio of the standard deviation to the mean of the CNT radius (calculated as an arithmetic average).

The electronic transport properties were studied using the non-equilibrium Green's function (NEGF) technique, within the Keldysh formalism [30] as implemented in the TranSIESTA and ATK codes [31,32]. The computed structures were treated as two-probe systems with the central scattering region sandwiched between fully relaxed semi-infinite source (left) and drain (right) copper electrode regions as shown in the insets of Fig.8 (a,d). Both interfaces between the $\mathrm{Cu}(100)$ surfaces and the CNT open ends were fully relaxed.

The current flowing through the scattering region was calculated from the corresponding Green's function and self-energies according to the Landauer-Büttiker formula:

$I(U)=\frac{2 \mathrm{e}}{\mathrm{h}} \int_{-\infty}^{+\infty} \mathrm{T}(\varepsilon, U)\left(\mathrm{f}_{\mathrm{l}}\left(\varepsilon-\mu_{\mathrm{l}}\right)-\mathrm{f}_{\mathrm{r}}\left(\varepsilon-\mu_{\mathrm{r}}\right)\right) \mathrm{d} \varepsilon$

where $\mu_{1(r)}$ is the electrochemical potential of the left (right) electrode and $f_{1(r)}$ is the corresponding electron distribution. $\mathrm{T}(\varepsilon, U)$ is the transmission coefficient of electrons incident at energy $\varepsilon$ through the central scattering region constituting the device under the bias voltage $\mathrm{U}=1 / \mathrm{e}\left(\mu_{1}-\mu_{\mathrm{r}}\right)$, given by the following formula:

$T(\varepsilon, U)=\left[\Gamma_{1}(\varepsilon) \mathrm{G}^{\mathrm{r}}(\varepsilon) \Gamma_{\mathrm{r}}(\varepsilon) \mathrm{G}^{\mathrm{a}}(\varepsilon)\right]$

where $G^{r(a)}$ is the retarded (advanced) Green's function of the central region and $\Gamma^{r(1)}$ is a matrix accounting for the coupling of the central region to the right (left) electrode. Hence, the conductance of the system can be calculated as:

$\mathrm{G}=\mathrm{G}_{\mathrm{o}} \operatorname{Tr}[T]$

where $G_{0}=\frac{2 e^{2}}{h}$ is the unit of quantum conductance. Consequently, the resistance $R$ is expressed as $1 / \mathrm{G}$. To calculate the transmission we used 30 points along the arc part of the complex contour, 10 on the line of the contour and 16 poles. The complex energy contour was always set to a value below the lowest energy in the energy spectrum of each system. The small finite complex part of the real energy contour was set to $10^{-6} \mathrm{Ry}$. The energy 
window was chosen in the range of $(-2,2) \mathrm{eV}$ and the number of points on the close-to-real axis part of the contour in the voltage bias window (n) was chosen to always meet the criterion $U / \mathrm{n}=0.01 \mathrm{eV}$.

To get a wider perspective on how interhalogens influence the electrical properties of CNTs, we have compared our results with a commonly used similar doping agent: iodine [14, 33, 34].

\section{Results and Discussion}

\subsection{Structure and Chemistry}

As-made material has a very porous network of loosely bound CNTs due to the fact that it is formed from a CNT aerogel synthesized in the furnace (Fig. 2a) [35, 36]. We wanted to find out how dopant treatment affects the microstructure of the material. The results show that, soaking in DCM (interhalogen compound solvent) causes an increase in packing density of the material (Fig. 2b).

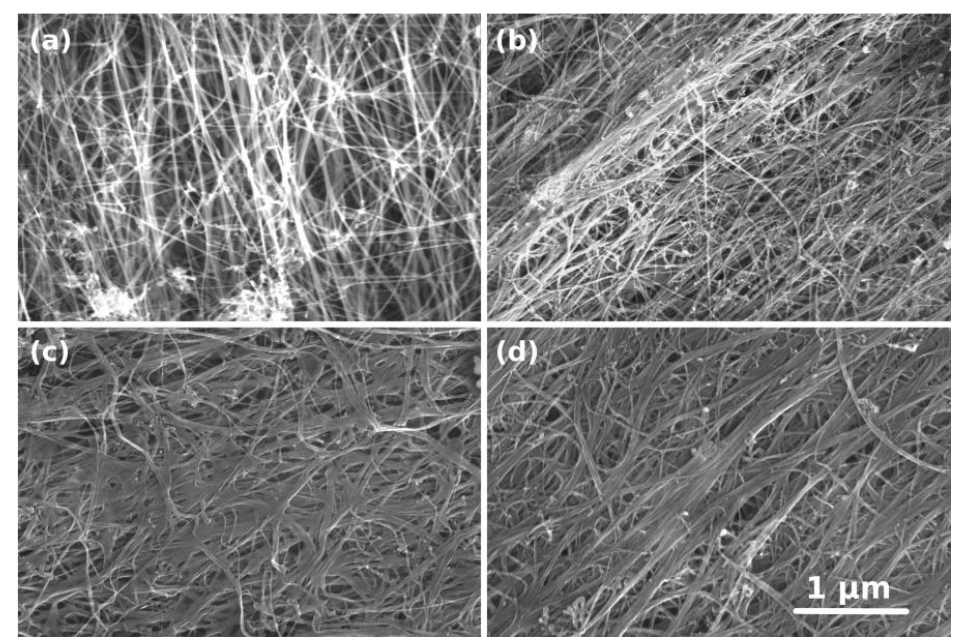

Fig. 2 SEM micrographs of CNT films. (a) as-made (b) treated with DCM, (c) treated with iodine monochloride in DCM, (d) treated with iodine monobromide in DCM.

Exposure of directly-spun material to volatile solvents (e.g. acetone, ethanol, DCM) is known to cause capillary-induced densification [37]. However, the observed densification is much 
more notable when interhalogen compounds in DCM are the medium, in which the CNT films are treated. Addition of both iodine monochloride and iodine monobromide in DCM brought the individual CNTs and their bundles much closer to each other (Fig. 2c,d). Supporting evidence is given by the contrast in the observed SEM micrographs. Doped samples do not have areas of high-contrast which is seen in as-made or DCM-only densified material. Such high contrast can be indicative of charging of the material, and thus poor electron propagation. These features cannot be found in the micrographs of ICl- and IBrdoped material. As will be shown later, that is indeed the case and doping of the CNT films by interhalogen compounds improves the electron transport to a large extent.
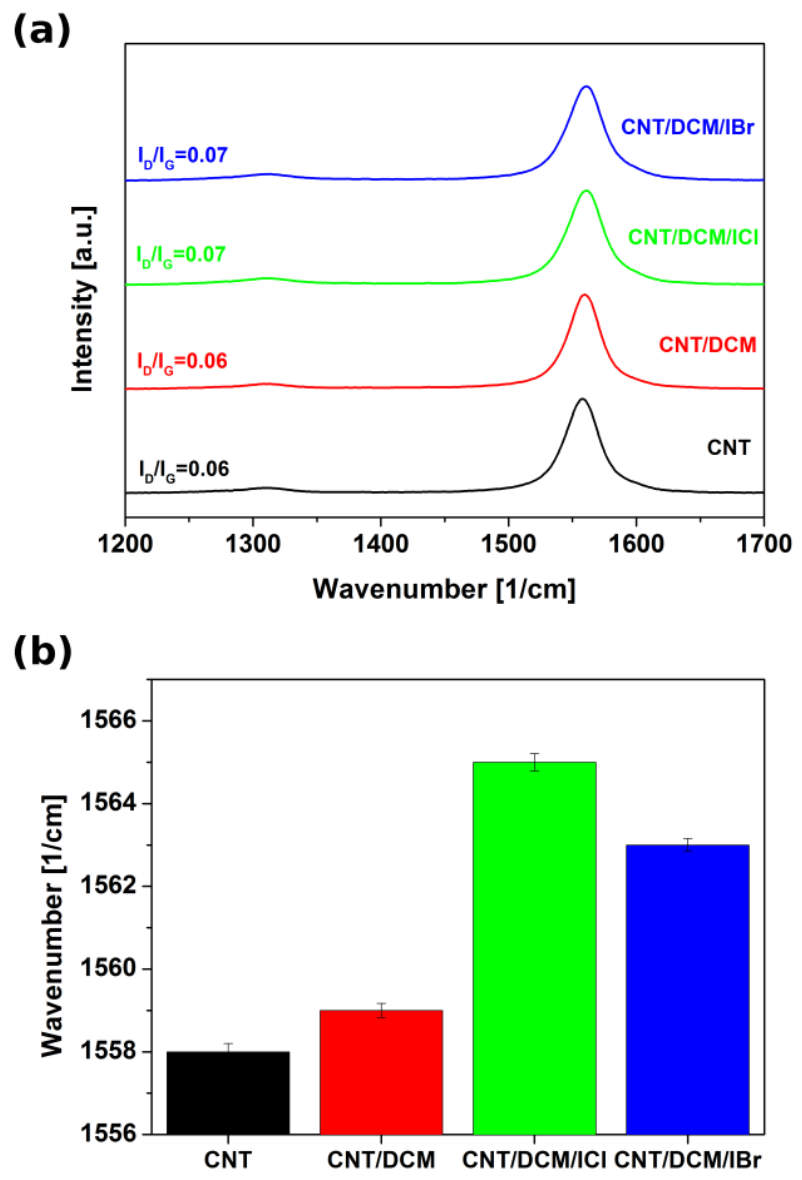

Fig. 3 (a) Raman spectra of as-made and treated CNT films. (b) position of the G-peak in the Raman spectra of as-made and treated CNT films. 
Furthermore, we carried out Raman spectroscopy of as-made and treated CNT films and compared the results with the DFT calculations of halogen doped CNTs to get information about the chemical structure of the material (Fig. 3a). The intensity ratio $\mathrm{I}_{\mathrm{D}} / \mathrm{I}_{\mathrm{G}}$ of the starting material showed that it was highly pure with only slight functionalization of the constituent CNTs. Next, densification of the material by soaking in DCM did not cause any change to the intensity of the peaks. This indicates that capillary-induced densification only restructures the material by improving its packing. Exposure of the CNT films to interhalogen compounds gave only a negligible increase in $\mathrm{I}_{\mathrm{D}} / \mathrm{I}_{\mathrm{G}}$, which suggests that physisorption dominates rather than chemical functionalization. This is beneficial because such an effect preserves the highly conductive network of $\mathrm{sp}^{2}$ carbon atoms and does not disrupt it with unnecessary exohedral bonds.

Table 1. DFT-calculated structural parameters, CV and band gap energies of isolated XY $(\mathrm{X}=\mathrm{I}, \mathrm{Y}=\mathrm{I}, \mathrm{Cl}, \mathrm{Br})$ interhalogen compounds and halogen doped CNTs. The results are compared to available experimental and other theoretical values.

\begin{tabular}{|c|c|c|c|c|c|}
\hline CNT & $\begin{array}{c}\text { Interhalogen } \\
\text { compound, } \\
\text { XY }\end{array}$ & $\begin{array}{c}\text { X-Y Bond length } \\
(\AA)\end{array}$ & $\begin{array}{c}\text { XY-CNT } \\
\text { Equilibrium } \\
\text { distance }(\AA)\end{array}$ & $\mathrm{CV}$ & $\mathrm{E}_{\text {gap, }}(\mathrm{eV})$ \\
\hline \multirow{3}{*}{---- } & $\mathrm{I}_{2}$ & $2.6895(2.666[38])$ & \multirow{3}{*}{---- } & \multirow{3}{*}{----} & \multirow{3}{*}{----} \\
\hline & $\mathrm{IBr}$ & $2.4923(2.47[39])$ & & & \\
\hline & $\mathrm{ICl}$ & $2.3482(2.3207[38])$ & & & \\
\hline \multirow{4}{*}{$(5,5)$} & ---- & --- & ---- & 0.0000 & 0 \\
\hline & $\mathrm{I}_{2}$ & 2.7105 & $3.1336(3.27[40])$ & 0.0060 & $0(0[40])$ \\
\hline & $\mathrm{IBr}$ & 2.5150 & $3.0294(3.07[40])$ & 0.0057 & $0(0.02[40])$ \\
\hline & $\mathrm{ICl}$ & 2.3736 & $2.9738(3.18[40])$ & 0.0048 & $0(0[40])$ \\
\hline \multirow{4}{*}{$(10,0)$} & ---- & ---- & ---- & 0.0003 & 0.790 \\
\hline & $\mathrm{I}_{2}$ & 2.6962 & 3.1801 & 0.0049 & 0.356 \\
\hline & $\mathrm{IBr}$ & 2.4968 & 3.0845 & 0.0045 & 0.397 \\
\hline & $\mathrm{ICl}$ & 2.3501 & 3.0320 & 0.0047 & 0.432 \\
\hline
\end{tabular}

In support of this conclusion our DFT calculations clearly indicate that halogen molecules create non-covalent bonding with CNTs, which is in agreement with earlier observations [33, 40-42] and the experimental results of this study. The distance between the CNTs and interhalogen compounds is around $3 \AA$ for all the cases considered (Table 1$)$. The iodine and interhalogen bond lengths near the CNTs are slightly elongated in comparison to the isolated 
case. The metallic CNT is more reactive than its semiconducting counterpart and therefore induces larger changes in the halogen compound structures [43]. Simultaneously the CNT structures are less affected by their interaction with the halogens than is observed when covalently functionalised [44]. The coefficient of CNT radius variation, which is a standardized measure of the change in nanotube shape, varies between only 0.0045 and 0.0060 and is an order of magnitude smaller than in the case of covalent functionalization [45].

Analysis of the position of the G-peak in the Raman spectra can give information about the type of doping caused by a particular treatment. In our case we observed a blue-shift in the Gpeak, which is indicative of electron transfer from the CNTs to the dopants [46, 47]. Electronegative iodine in the form of $\mathrm{I}^{+}$is highly electrophilic and strongly withdraws electrons from the CNTs, effectively improving hole mobility. The size of the blue-shifts were found to be consistent with the reported electron affinities of $\mathrm{ICl}$ and $\mathrm{IBr}$ [40].

Surprisingly, interaction between the CNTs and the halogen molecules induces changes in electronic properties similar to that found from covalent bonding [44]. $\mathrm{I}_{2}, \mathrm{IBr}$ and $\mathrm{ICl}$ introduce additional impurity bands near the Fermi level (Figure 4), significantly modifying the band gap energy in the case of the semiconducting CNT (Table 1). In the case of $\mathrm{I}_{2}$ physisorbed to the $(10,0) \mathrm{CNT}$, the band gap was reduced by $55 \%$ compared to the pure $(10,0)$ CNT. As expected, the band gap reduction agrees with the electron donating order of the halogens: $\mathrm{I}_{2}>\mathrm{IBr}>\mathrm{ICl}$. This trend is consistent with a previous observation of the metallization of smaller semiconducting nanotube induced by halogen interactions [40]. On the other hand, physisorption of halogen compounds to the metallic $(5,5) \mathrm{CNT}$ does not significantly change the electronic properties of the system.

To reveal the origin of the impurity bands we plotted the projected density of states (PDOS) for all the systems considered as shown in Fig. 4. 

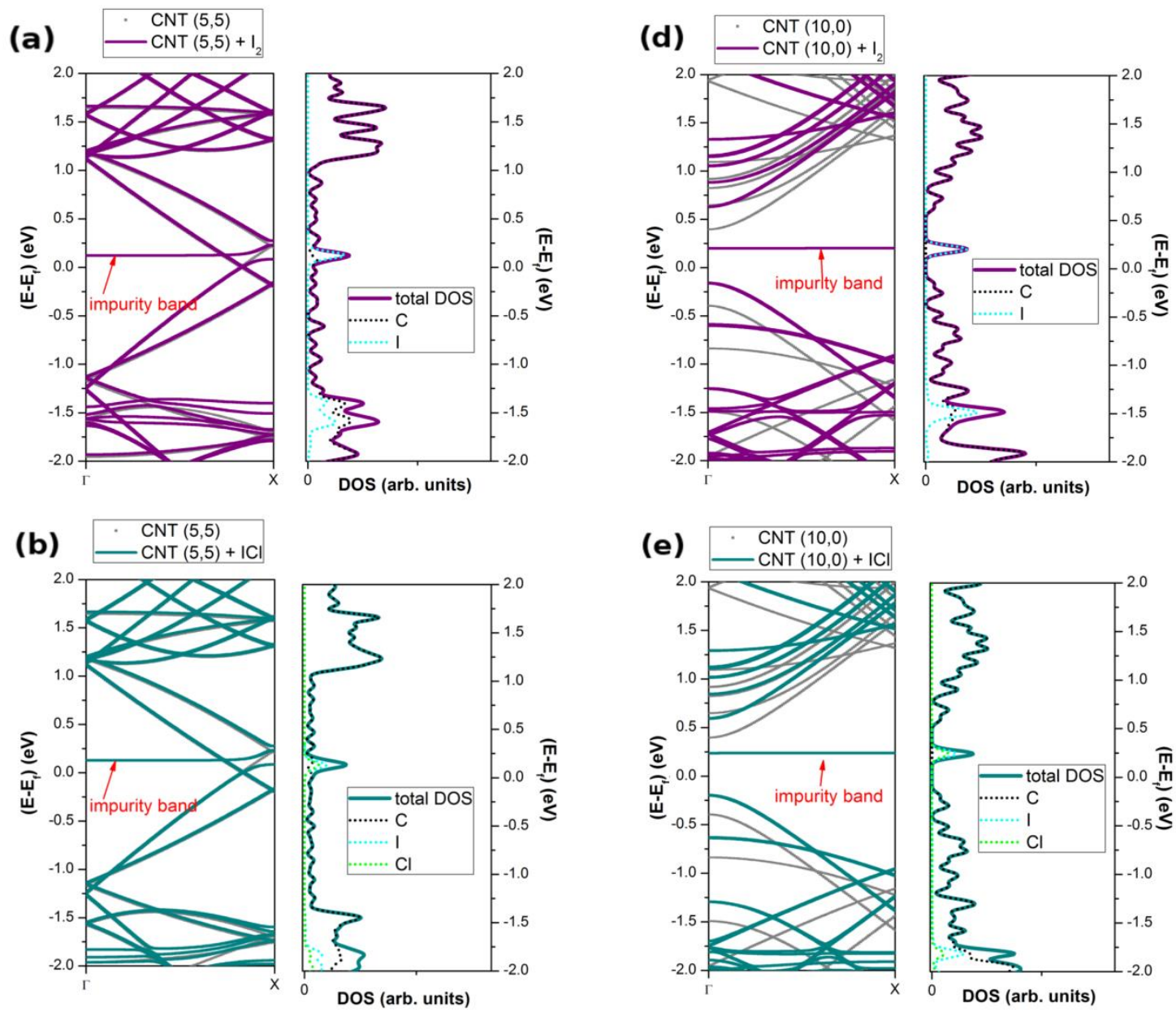

(c) $\quad$ CNT $(5,5)$
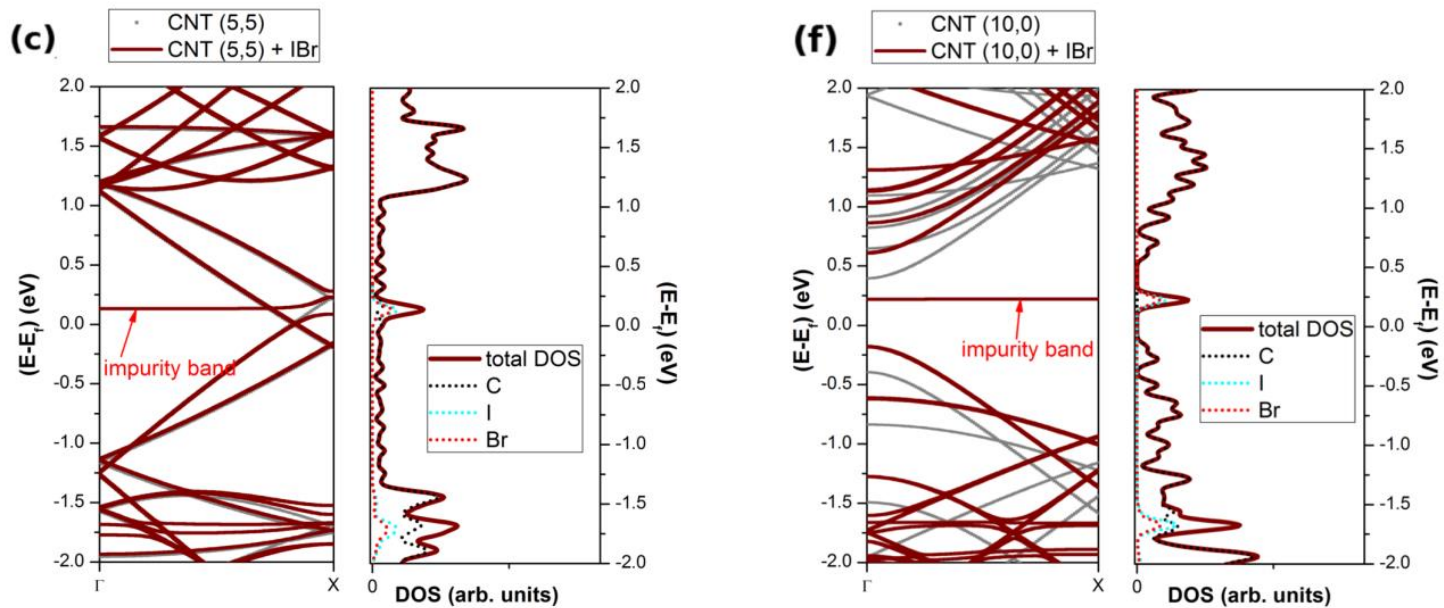

Fig. 4 DFT-calculated electronic band structures of the functionalised CNTs along $\Gamma \rightarrow X$ of the Brillouin zone together with the projected density of states (PDOS) of functionalized CNTs. For comparison, the band structures of pure $(5,5)$ and $(10,0)$ CNTs are denoted by grey lines. The Fermi energy is set to zero. Impurity bands originating from the presence on halogen molecules are labelled. 
The main contribution to the impurity bands near the Fermi level comes from the interhalogen compounds. Also, to a smaller extent, carbon atoms from the CNT backbone contribute to the impurity band. As expected, the latter contribution is more pronounced for the metallic CNT than for the semiconducting one. However, analysis of the chemical nature of the impurity bands clearly shows that for both types of CNT and all interhalogen compounds, the chemical nature of these bands is very similar. It is important to note that the additional impurity bands can open new conducting channels and contribute electrons to existing ones.

As confirmed by EDX (Fig. 5), the interhalogen compounds can be readily physisorbed onto the CNT films. After treatment with ICl, the CNT film contained $4.4 \mathrm{wt} \%$ of chlorine and 1.9 wt $\%$ of iodine. Exposure to $\mathrm{IBr}$ resulted in $5.8 \mathrm{wt} \%$ of bromine and $2.2 \mathrm{wt} \%$ of iodine in the modified CNT film. Densification by DCM did not cause any change as expected.

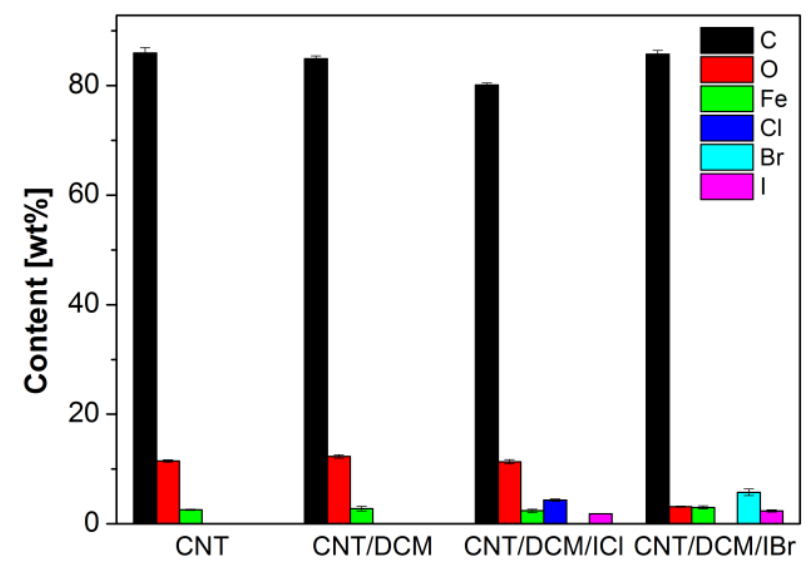

Fig. 5 Composition of the as-made and halogen treated CNT films from EDX measurements. 
3.2 Thermal Stability

(a)

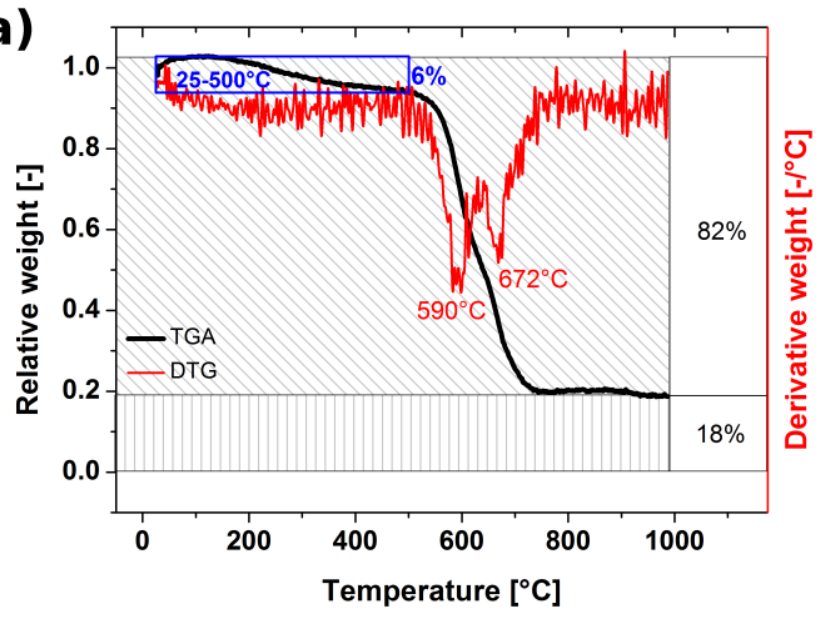

(b)

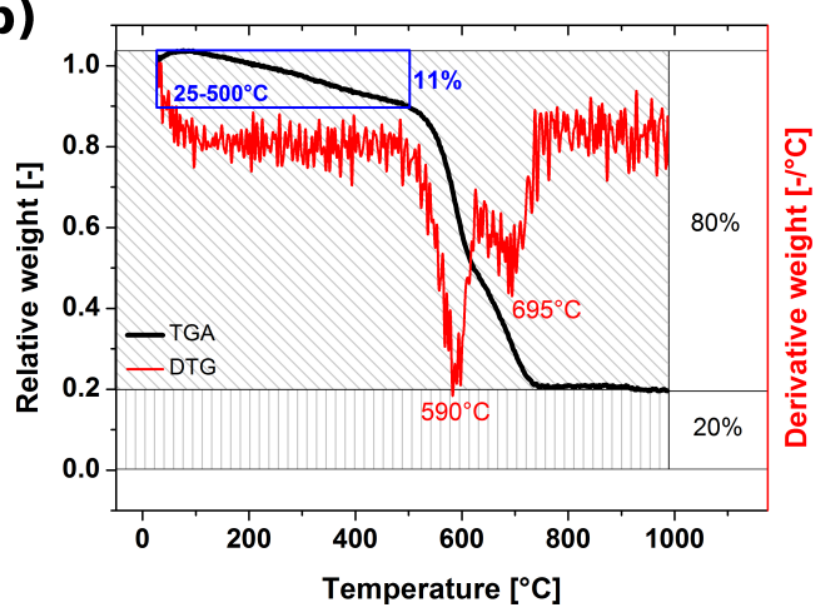

(c)

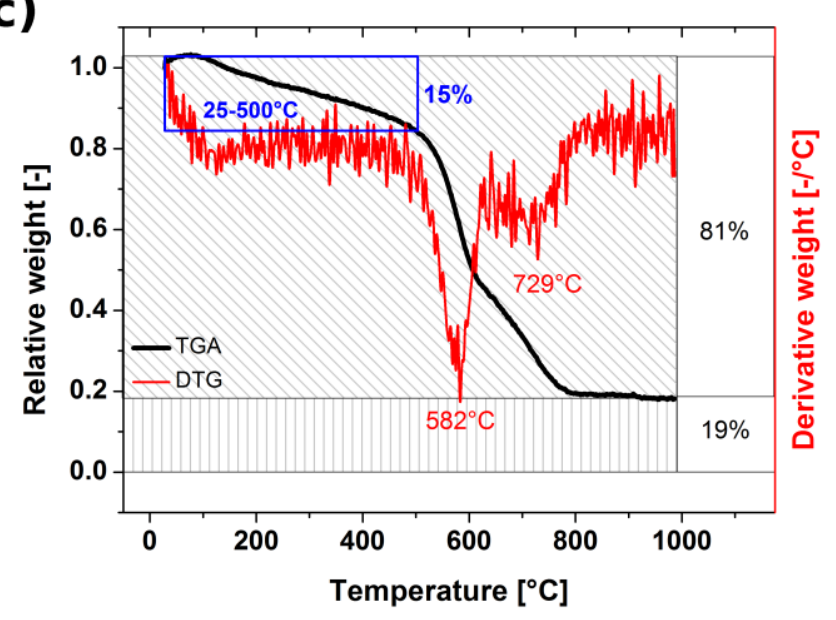

Fig. 6 Thermograms of the CNT films. (a) as-made (b) treated with iodine monochloride in dichloromethane, (c) treated with iodine monobromide in dichloromethane. 
Thermogravimetric analysis (TGA) (Fig. 6) showed that the as-made material is of moderate thermal stability. Between r.t. and $500^{\circ} \mathrm{C}$ we observed a $6 \%$ weight loss, which can be explained by desorption of low molecular weight polyaromatic contaminants commonly present in the material [48]. In the same temperature range, other non-CNT contaminants with inferior thermal properties such as amorphous carbon can be combusted. The main thermal degradation of the CNTs takes place between $500^{\circ} \mathrm{C}$ and $700^{\circ} \mathrm{C}$. The thermogram of the as-made CNT film (Fig. 6 a) illustrates the dual nature of the material, wherein we have two main CNT population types with maximum rate of decomposition temperatures at $590^{\circ} \mathrm{C}$ and $672^{\circ} \mathrm{C}$. Addition of iodine monochloride (Fig. 6b) and iodine monobromide (Fig. 6c) correctly reflects the shapes of the thermograms in the typical range for removal of functional groups [49]. The weight loss between r.t. and $500^{\circ} \mathrm{C}$ is $11 \%$ for the ICl- and $15 \%$ for the IBrdoped CNT films. The atomic mass of bromine is higher than that of chlorine, and so it has got a larger contribution to the recorded weight.

\subsection{Electrical Properties}

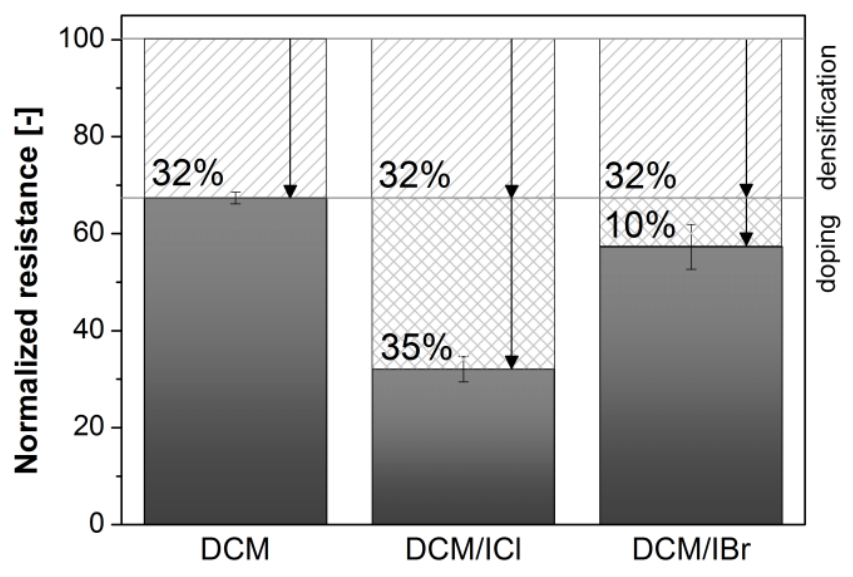

Fig. 7 The influence of interhalogen treatment on electrical resistance.

Finally, we investigated the effect of interhalogen addition on the electrical properties of the CNT films. Halogens $[14,33]$ and interhalogen compounds $[8,40]$ have a powerful doping 
effect on nanocarbon materials. In our case, the highest reduction in electrical resistance (67\%) was when iodine monochloride was employed. Doping by iodine monobromide accounted for a $42 \%$ reduction in electrical resistance. Because some of this effect is also due to capillary-induced densification, we have also measured the influence of the addition of neat DCM, which was found to produce as much as a $32 \%$ reduction and so this should be subtracted from the observed performance of interhalogen agents dissolved in this medium. DCM causes a notable reduction in sample dimensions, which brings the CNTs and their bundles closer together and so improves the electron transport. The fact that $\mathrm{ICl}$ is a stronger doping agent than $\mathrm{IBr}$ follows our previous observation that it has a higher electron affinity than $\mathrm{IBr}$ (Fig. 2b). It is important to note the reason for using a normalized resistance and not absolute values for this study. The CNT films produced by the direct-spinning method often have a non-uniform cross-section due to their aerogel nature. This brings a significant error into precise determination of resistivity in units of $\mathrm{S} / \mathrm{cm}$. To have a reliable comparison between different treatments when undensified CNT films are the starting material, it is often necessary to normalize the values to the initial resistance measured at room temperature $[8$, 16].

To better understand the physical mechanism governing the observed reduction in electrical resistance we have performed transport calculations on interhalogen doped CNTs and compared them with the iodine reference. As in the experiment, $\mathrm{Cu}$ electrodes were used. Copper is a metal rather weakly interacting with the CNT and this preserves some intrinsic characteristics of the CNT's electronic structure when contact is created [50], but also causes some changes. Charge transfer between the metallic electrodes and the CNT produces band bending which enables the CNT's valence band edge to align with the Fermi level of the electrodes [51]. Due to coupling with the Cu electrodes, armchair CNTs exhibit a reduced $\mathrm{sp}^{2}$ hybridization configuration resulting in a smaller conductance than with CNT electrodes [52]. 
(a)

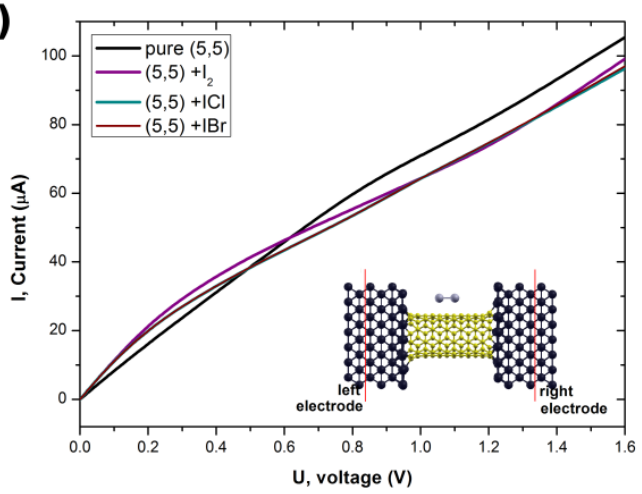

(b)

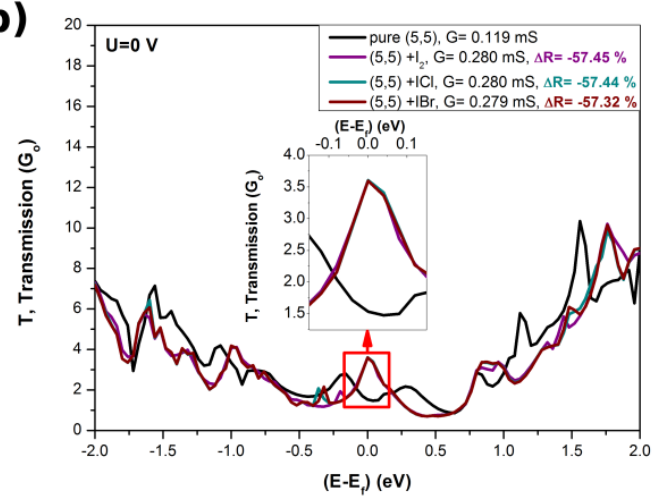

(c)

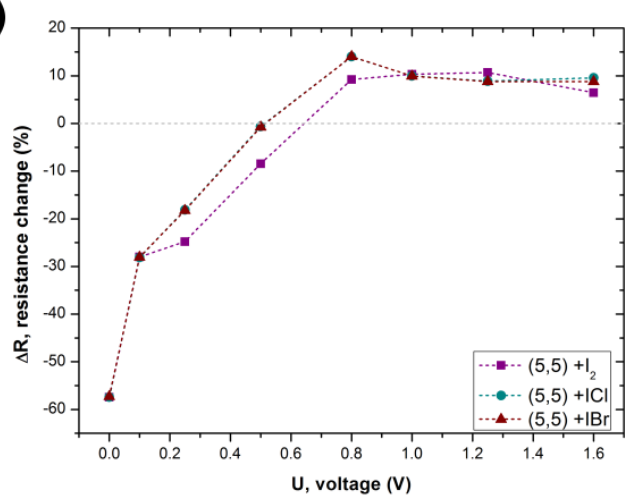

(d)

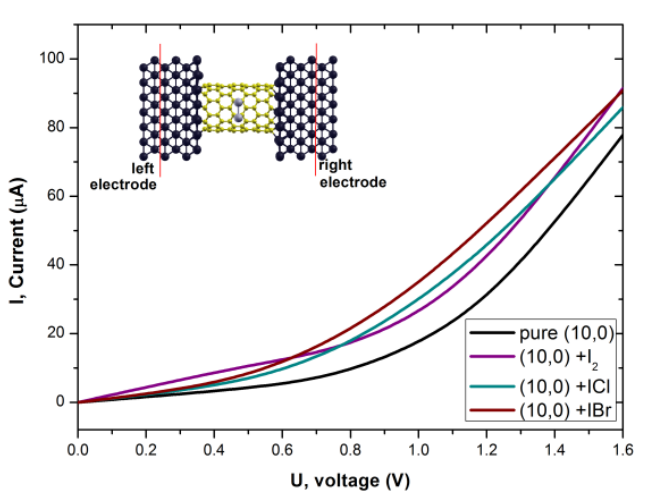

(e)

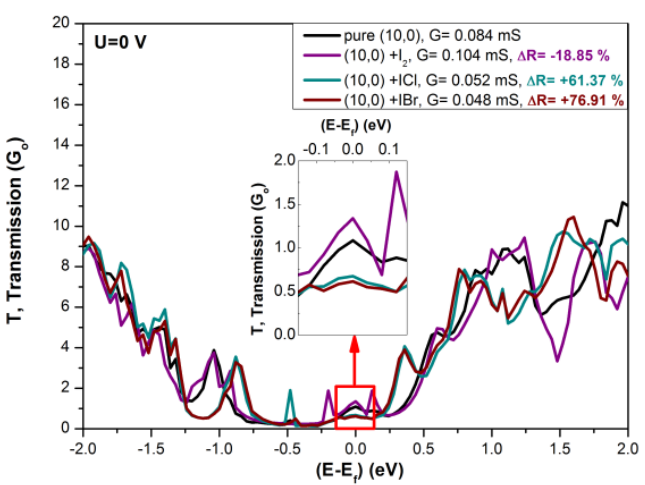

(f)

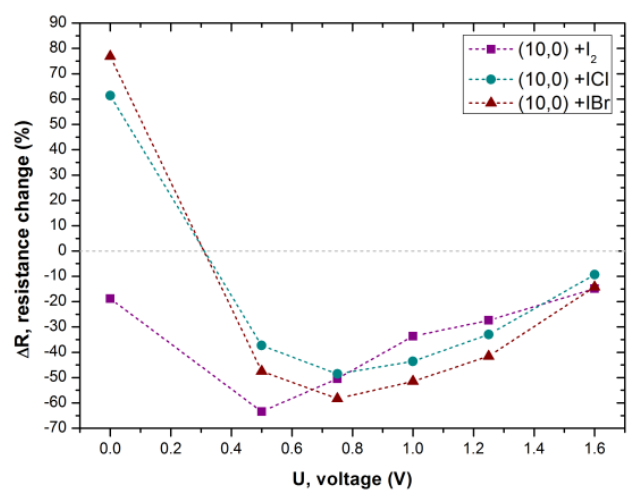

Fig. 8 Calculated electrical properties of pure and doped metallic $(5,5)$ and semiconducting $(10,0)$ CNTs sandwiched between Cu electrodes: $(\mathrm{a}, \mathrm{d})$ current-voltage characteristics, $(\mathrm{b}, \mathrm{e})$ zero-bias transmission spectra, $(\mathrm{c}, \mathrm{f})$ resistance change with applied voltage, respectively.

The current-voltage characteristics are approximately linear although non-Ohmic behavior is seen in the high bias regime [50]. On the other hand, semiconducting CNTs sandwiched between $\mathrm{Cu}$ electrodes become metallic [52] due to the breaking of the mirror symmetry of the nanotube wave functions induced by the localized perturbation of the leads [51]. Metallic 
contacts introduce additional states, the so-called metal-induced gap states [53, 54], around the Fermi level in an energy region where the DOS of the pure semiconducting CNT does not have any non-zero values. Since transport through the system is ballistic, the transmission coefficient at the Fermi level is correlated with the number of bands. Therefore, the conductance of a semiconducting $\mathrm{CNT}$ connected to $\mathrm{Cu}$ electrodes is not only higher than of metal-free semiconducting CNTs but also the characteristic critical voltage for the non-zero current cannot be observed [55].

Bearing in mind this description of the transport properties of a pure CNT connected to $\mathrm{Cu}$ electrodes, let us focus on changes induced by halogen doping. The calculated currentvoltage characteristics of $(5,5)$ and $(10,10)$ CNTs doped with halogen compounds compared to the $I(U)$ characteristics of the pristine CNTs are depicted in Fig. 8a and Fig. 8d, respectively. The transmission coefficients at the Fermi level under zero bias voltage applied to all $(5,5)$ halogen-doped systems are higher by $\sim 135 \%$ than that of the undoped $(5,5) \mathrm{CNT}$ (Fig. 8b). However, the resistance difference $\left(\Delta R=\left(R_{\text {doped }}-R_{\text {pure }}\right) / R_{\text {pure }}\right)$ quickly diminishes with increasing applied voltage, being almost zero when $U \sim 0.6 \mathrm{~V}$ (Fig. 8c). For higher applied voltages the resistance of the halogen-doped system increases above the level of the resistance of the pure $(5,5) \mathrm{CNT}$. For the semiconducting nanotubes, which comprise $2 / 3$ of as-made single walled CNTs, the situation is quite different. Only the $I_{2}$ doped $(10,0)$ CNT has a transmission coefficient higher than that of the pure $(10,0)$ CNT under zero applied bias (by $\sim 23 \%$ ) (Fig 8e). Upon application of a finite voltage, the resistance of the $\mathrm{ICl}$ and $\mathrm{IBr}$ doped CNTs significantly decreases $(\Delta R=-49 \%$ for $\mathrm{ICl}$ and $\Delta R=-58 \%$ for $\mathrm{IBr})$ when compared with the undoped $(10,0)$ CNT until $U=0.75 \mathrm{~V}$ when it starts to increase again (Fig. 8f). The $\mathrm{I}_{2}$ doped $(10,0) \mathrm{CNT}$ follows the same trend but the maximum reduction in resistance $\Delta R=-63 \%$, is observed when $U=0.5 \mathrm{~V}$. It should be also noted that when the bias is small $(\mathrm{U} \leq 0.5 \mathrm{~V})$ the resistance reduction follows the same trend as band gap 
reduction: $\mathrm{I}_{2}>\mathrm{IBr}>\mathrm{ICl}$ (cf. Table 1). Clearly, the calculations confirm the experimental observations, and thus also show that halogen doping reduces the overall resistance of the CNT system.

\section{Conclusions}

In conclusion, all the above results confirm that interhalogen compounds such as iodine monochloride or iodine monobromide are potent doping agents for CNTs. The processing route is very straightforward and causes a permanent increase in the material's electrical conductivity without disrupting the somewhat fragile $\mathrm{sp}^{2}$ highly-conductive network of carbon atoms. Their addition results in simple, but stable physisorption rather than destructive chemical functionalization.

Furthermore, in addition to an improvement to the junction resistance caused by increased packing of the material, the doping agents themselves strongly withdraw electrons from CNTs, which results in evident p-doping of the material. Closer analysis using first principles calculations revealed that the halogen-doping agents react with metallic and semiconducting CNTs differently, and the latter are most affected. The proposed underlying mechanism is that an impurity band introduced by the doping agent lowers the band gap, which reduces the overall resistance of the CNT system.

The study demonstrates that interhalogen atoms are a viable alternative to iodine for doping carbon nanostructures. Their effect is permanent and does not diminish as with iodine, which, despite a favourable effect on conductivity, easily sublimes away with time.

\section{Acknowledgements}

D.J., K.Z.M. and K.K.K. acknowledge the European Research Council (under the Seventh Framework Program FP7/2007-2013, ERC grant agreement no 259061) and the Royal Society for financial support. We also thank the Interdisciplinary Centre for Mathematical and Computational Modelling at the University of Warsaw (Grant No. G47-5) and the 
Cambridge High Performance Computing Cluster for providing computer facilities. D.J. would also like to thank National Science Center, Poland (under the Polonez program, grant agreement UMO-2015/19/P/ST5/03799) and the European Union's Horizon 2020 research and innovation programme (Marie Skłodowska-Curie grant agreement 665778)

\section{References}

[1] M.-F. Yu, O. Lourie, M.J. Dyer, K. Moloni, T.F. Kelly, R.S. Ruoff, Strength and Breaking Mechanism of Multiwalled Carbon Nanotubes Under Tensile Load, Science 287(5453) (2000) 637-640.

[2] C. Lee, X. Wei, J.W. Kysar, J. Hone, Measurement of the Elastic Properties and Intrinsic Strength of Monolayer Graphene, Science 321(5887) (2008) 385.

[3] E. Pop, D. Mann, Q. Wang, K. Goodson, H. Dai, Thermal Conductance of an Individual Single-Wall Carbon Nanotube above Room Temperature, Nano Letters 6(1) (2006) 96-100.

[4] E.T. Thostenson, C. Li, T.-W. Chou, Nanocomposites in context, Composites Science and Technology 65(3-4) (2005) 491-516.

[5] A.A. Balandin, S. Ghosh, W. Bao, I. Calizo, D. Teweldebrhan, F. Miao, C.N. Lau, Superior Thermal Conductivity of Single-Layer Graphene, Nano Letters 8(3) (2008) 902-907.

[6] S. Hong, S. Myung, Nanotube Electronics: A flexible approach to mobility, Nat Nano 2(4) (2007) 207-208.

[7] S. Stankovich, D.A. Dikin, G.H.B. Dommett, K.M. Kohlhaas, E.J. Zimney, E.A. Stach, R.D. Piner, S.T. Nguyen, R.S. Ruoff, Graphene-based composite materials, Nature 442(7100) (2006) 282-286.

[8] D. Janas, A.P. Herman, S. Boncel, K.K.K. Koziol, lodine monochloride as a powerful enhancer of electrical conductivity of carbon nanotube wires, Carbon 73 (2014) 225-233.

[9] G.J. Brady, A.J. Way, N.S. Safron, H.T. Evensen, P. Gopalan, M.S. Arnold, Quasi-ballistic carbon nanotube array transistors with current density exceeding $\mathrm{Si}$ and GaAs, Science Advances 2(9) (2016).

[10] R.S. Lee, H.J. Kim, J.E. Fischer, A. Thess, R.E. Smalley, Conductivity enhancement in single-walled carbon nanotube bundles doped with K and Br, Nature 388(6639) (1997) 255-257.

[11] M. Schmid, C. Goze-Bac, S. Krämer, S. Roth, M. Mehring, C. Mathis, P. Petit, Metallic properties of Li-intercalated carbon nanotubes investigated by NMR, Physical Review B 74(7) (2006) 073416.

[12] C. Wang, K. Ryu, A. Badmaev, N. Patil, A. Lin, S. Mitra, H.-S.P. Wong, C. Zhou, Device study, chemical doping, and logic circuits based on transferred aligned single-walled carbon nanotubes, Applied Physics Letters 93(3) (2008) 033101.

[13] K.S. Mistry, B.A. Larsen, J.D. Bergeson, T.M. Barnes, G. Teeter, C. Engtrakul, J.L. Blackburn, nType Transparent Conducting Films of Small Molecule and Polymer Amine Doped Single-Walled Carbon Nanotubes, ACS Nano 5(5) (2011) 3714-3723.

[14] D. Janas, S. Boncel, K.K.K. Koziol, Electrothermal halogenation of carbon nanotube films, Carbon 73 (2014) 259-266.

[15] W. Zhou, J. Vavro, N.M. Nemes, J.E. Fischer, F. Borondics, K. Kamarás, D.B. Tanner, Charge transfer and Fermi level shift in \$p\$-doped single-walled carbon nanotubes, Physical Review B 71(20) (2005) 205423.

[16] D. Janas, A.C. Vilatela, K.K.K. Koziol, Performance of carbon nanotube wires in extreme conditions, Carbon 62 (2013) 438-446.

[17] L.S. Panchakarla, A. Govindaraj, C.N.R. Rao, Boron- and nitrogen-doped carbon nanotubes and graphene, Inorganica Chimica Acta 363(15) (2010) 4163-4174.

[18] Y.L. Li, I.A. Kinloch, A.H. Windle, Direct spinning of carbon nanotube fibers from chemical vapor deposition synthesis, Science 304(5668) (2004) 276-8. 
[19] K. Koziol, J. Vilatela, A. Moisala, M. Motta, P. Cunniff, M. Sennett, A. Windle, High-Performance Carbon Nanotube Fiber, Science 318(5858) (2007) 1892-1895.

[20] L. Min-Yang, Y. Mingchia, V. Emily, N. Kyle, V. Arda, L. Richard, Analysis of variance on thickness and electrical conductivity measurements of carbon nanotube thin films, Measurement Science and Technology 27(9) (2016) 095004.

[21] P. Hohenberg, W. Kohn, Inhomogeneous Electron Gas, Physical Review 136(3B) (1964) B864B871.

[22] W. Kohn, L.J. Sham, Self-Consistent Equations Including Exchange and Correlation Effects, Physical Review 140(4A) (1965) A1133-A1138.

[23] P. Ordejón, E. Artacho, J.M. Soler, Self-consistent order-\$N\$ density-functional calculations for very large systems, Physical Review B 53(16) (1996) R10441-R10444.

[24] J.M. Soler, E. Artacho, J.D. Gale, A. Garcia, J. Junquera, P. Ordejon, D. Sanchez-Portal, The SIESTA method for ab initio order-N materials simulation, J Phys-Condens Mat 14(11) (2002) 2745-2779.

[25] J.P. Perdew, K. Burke, M. Ernzerhof, Generalized Gradient Approximation Made Simple, Physical Review Letters 77(18) (1996) 3865-3868.

[26] N. Troullier, J.L. Martins, Efficient pseudopotentials for plane-wave calculations, Physical Review B 43(3) (1991) 1993-2006.

[27] L. Kleinman, D.M. Bylander, Efficacious Form for Model Pseudopotentials, Physical Review Letters 48(20) (1982) 1425-1428.

[28] S. Grimme, Semiempirical GGA-type density functional constructed with a long-range dispersion correction, Journal of Computational Chemistry 27(15) (2006) 1787-1799.

[29] H.J. Monkhorst, J.D. Pack, Special points for Brillouin-zone integrations, Physical Review B 13(12) (1976) 5188-5192.

[30] S. Datta, Electronic transport in mesoscopic systems, Cambridge University Press, Cambridge ; New York, 1995.

[31] M. Brandbyge, J.-L. Mozos, P. Ordejón, J. Taylor, K. Stokbro, Density-functional method for nonequilibrium electron transport, Physical Review B 65(16) (2002) 165401.

[32] Atomistix ToolKit, Copenhagen, Denmark, 2015.

[33] Y. Zhao, J. Wei, R. Vajtai, P.M. Ajayan, E.V. Barrera, lodine doped carbon nanotube cables exceeding specific electrical conductivity of metals, Scientific Reports 1 (2011) 83.

[34] L. Fan, X. Xu, A simple strategy to enhance electrical conductivity of nanotube-conjugate polymer composites via iodine-doping, RSC Advances 5(95) (2015) 78104-78108.

[35] D. Janas, K.K. Koziol, Rapid electrothermal response of high-temperature carbon nanotube film heaters, Carbon 59 (2013) 457-463.

[36] T.Q. Tran, Z. Fan, A. Mikhalchan, P. Liu, H.M. Duong, Post-Treatments for Multifunctional Property Enhancement of Carbon Nanotube Fibers from the Floating Catalyst Method, ACS Applied Materials \& Interfaces 8(12) (2016) 7948-7956.

[37] D. Janas, A. Cabrero-Vilatela, J. Bulmer, L. Kurzepa, K.K. Koziol, Carbon nanotube wires for hightemperature performance, Carbon 64 (2013) 305-314.

[38] L.E. Sutton, Table of interatomic distances and configuration in molecules and ions (Supplement 1956-1959) The Chemical Society 1965.

[39] P.B. Saxena, Chemistry of Interhalogen Compounds, Discovery, New Delhi, 2007.

[40] S. Ghosh, S.R.K.C.S. Yamijala, S.K. Pati, C.N.R. Rao, The interaction of halogen molecules with SWNTs and graphene, RSC Advances 2(3) (2012) 1181-1188.

[41] D.A. Britz, A.N. Khlobystov, Noncovalent interactions of molecules with single walled carbon nanotubes, Chemical Society Reviews 35(7) (2006) 637-659.

[42] Z. Wang, L. Wang, Z. Shi, J. Lu, Z. Gu, Z. Gao, Tuning of hole doping level of iodine-encapsulated single-walled carbon nanotubes by temperature adjustment, Chemical Communications (29) (2008) 3429-3431.

[43] P.A. Denis, F. Iribarne, On the applicability of cluster models to study the chemical reactivity of carbon nanotubes, Journal of Computational Chemistry 32(11) (2011) 2397-2403. 
[44] K.Z. Milowska, J.A. Majewski, Functionalization of carbon nanotubes with $-\mathrm{CHn},-\mathrm{NHn}$ fragments, $-\mathrm{COOH}$ and -OH groups, The Journal of Chemical Physics 138(19) (2013) 194704.

[45] K.Z. Milowska, Influence of Carboxylation on Structural and Mechanical Properties of Carbon Nanotubes: Composite Reinforcement and Toxicity Reduction Perspectives, The Journal of Physical Chemistry C 119(47) (2015) 26734-26746.

[46] W.S. Jang, S.S. Chae, S.J. Lee, K.M. Song, H.K. Baik, Improved electrical conductivity of a noncovalently dispersed graphene-carbon nanotube film by chemical p-type doping, Carbon 50(3) (2012) 943-951.

[47] D. Janas, S. Boncel, A.A. Marek, K.K. Koziol, A facile method to tune electronic properties of carbon nanotube films, Materials Letters 106 (2013) 137-140.

[48] S. Boncel, R.M. Sundaram, A.H. Windle, K.K.K. Koziol, Enhancement of the Mechanical Properties of Directly Spun CNT Fibers by Chemical Treatment, ACS Nano 5(12) (2011) 9339-9344.

[49] S. Mallakpour, A. Abdolmaleki, S. Borandeh, I-Phenylalanine amino acid functionalized multi walled carbon nanotube (MWCNT) as a reinforced filler for improving mechanical and morphological properties of poly(vinyl alcohol)/MWCNT composite, Progress in Organic Coatings 77(11) (2014) 1966-1971.

[50] F. Gao, J. Qu, M. Yao, Electronic structure and contact resistance at an open-end carbon nanotube and copper interface, Applied Physics Letters 96(10) (2010) 102108.

[51] M.B. Nardelli, J.L. Fattebert, J. Bernholc, $\$ O(N) \$$ real-space method for \textit\{ab initio\} quantum transport calculations: Application to carbon nanotube $\backslash$ char21\{\}metal contacts, Physical Review B 64(24) (2001) 245423.

[52] G.-x. Wu, Q.-y. Meng, C.-y. Wang, I-V curves of carbon nanotubes with copper contacts using non-equilibrium Green's function method, Physica E: Low-dimensional Systems and Nanostructures 44(1) (2011) 146-151.

[53] J. Tersoff, Schottky Barrier Heights and the Continuum of Gap States, Physical Review Letters 52(6) (1984) 465-468.

[54] J. Tersoff, Schottky barriers and semiconductor band structures, Physical Review B 32(10) (1985) 6968-6971.

[55] K.Z. Milowska, Mechanical and Electrical Properties of Covalently Functionalized Carbon Nanotubes and Graphene Layers, University of Warsaw, Warsaw, 2013. 